\title{
Chemotherapy Toxicity Profile in Adjuvant Treated Colorectal Carcinoma Patients
}

\author{
Nagy Samy Gobran, Mohammed Reda Kelany, Mohammed Abdallah Fathy* \\ Clinical Oncology \& Nuclear Medicine Department, Ain Shams University, Cairo, Egypt \\ Email: ${ }^{\star}$ fetieh1987@gmail com
}

How to cite this paper: Gobran, N.S., Kelany, M.R. and Fathy, M.A. (2020) Chemotherapy Toxicity Profile in Adjuvant Treated Colorectal Carcinoma Patients. Journal of Cancer Therapy, 11, 74-87. https://doi.org/10.4236/jct.2020.112007

Received: February 3, 2020

Accepted: February 25, 2020

Published: February 28, 2020

Copyright $\odot 2020$ by author(s) and Scientific Research Publishing Inc. This work is licensed under the Creative Commons Attribution International License (CC BY 4.0).

http://creativecommons.org/licenses/by/4.0/ (c) (i) Open Access

\begin{abstract}
Background: Colorectal cancer (CRC) is the third most common cancer and the fourth leading cause of cancer-related deaths in the world. The treatment of stage III CRC consists of surgery followed by adjuvant chemotherapy with either single agent capecitabine or combination therapy consisting of FOLFOX or XELOX, all of which have been shown to be effective in improving disease-free survival (DFS) and overall survival (OS). However, toxicities acquired from chemotherapy can affect a cancer patient's quality of life and result in early treatment discontinuation. Common toxicities include hematological, gastrointestinal (GI), constitutional, dermatological, and neurological. Aim of the Work: The present work was aimed to assess and evaluate chemotherapy toxicities in adjuvant treatment in CRC patients and analyze certain factors that might increase chemotherapy toxicity. Patients and Methods: This is retrospective study included a total of 72 patients of colorectal carcinoma received adjuvant chemotherapy at Clinical Oncology Department, Ain Shams University. The study was conducted between Jan. 2012 and Jan. 2017. Results: We found that the most chemotherapy reported was neurological toxicity in (73.6\%), gastrointestinal symptoms (diarrhea $52.7 \%$, nausea $30.6 \%$, vomiting $27.8 \%$ \& oral mucositis $24 \%$ ), hematological toxicity (neutropenia $40.3 \%$, anemia $34.7 \%$, thrombocytopenia $12.5 \%$ ), fatigue $20.9 \%$, hepatic toxicity $18.1 \%$, dermatological toxicity $9.7 \%$ \& renal toxicity $5.6 \%$ ). older patients have significant incidence of neurological toxicity ( $\mathrm{p}$-value $=0.023)$ and fatigue ( $\mathrm{p}$-value $=0.038)$. females have significant incidence of anemia ( $p$-value $=0.017$ ). increase of oxaliplatin cumulative dose increase incidence of neurological toxicity $(\mathrm{p}$-value $=$ $0.024)$, thrombocytopenia ( $\mathrm{p}$-value $=0.007)$ and renal toxicity ( $\mathrm{p}$-value $=$ 0.030). Oxaliplatin containing regimens have high significant correlation with neurological toxicity ( $\mathrm{p}$-value $=0.000$ ) and capcitabine has high significant correlation with dermatological toxicity $(\mathrm{p}$-value $=0.000)$. Conclusion: The most overall toxicity reported during adjuvant treatment in CRC was neurological toxicity. Although a variety of adverse reactions were reported the treat-
\end{abstract}


ment regimens were tolerated but we should take care of factors that may increase certain toxicity.

\section{Keywords}

Chemotherapy Toxicity, Adjuvant Chemotherapy, CRC

\section{Introduction}

Colorectal cancer is the third most common cancer and the second leading cause of cancer death in the United States [1]. Approximately 140,250 new cases of colorectal cancer diagnosed in 2018 in the US, and nearly 50,630 patients will die from this disease [1]. Colorectal cancer is also a significant global public health issue, with 1.2 million cases diagnosed and 600,000 associated deaths each year [2]. At the time of initial diagnosis, nearly $40 \%$ of colorectal cancer patients present with localized disease [1]. Five-year survival rates vary with the stage at initial diagnosis, reflecting the risk of cancer recurrence after curative surgical resection of the primary tumor [3].

A significant proportion of stage III colon cancer patients who undergo potentially curative surgical resection develop disease recurrence secondary to clinically occult micro metastatic disease present at the time of surgery. Adjuvant chemotherapy administered after curative surgical resection has the potential to eradicate micro metastatic disease, thereby increasing the chance of cure. The clinical benefits of adjuvant chemotherapy, with respect to prolonging overall survival (OS), have been clearly demonstrated in stage III colon cancer [4] [5]. For nearly 15 years, oxaliplatin-based chemotherapy for 6 months, with FOLFOX (folinic acid, fluorouracil [5-FU], oxaliplatin) or XELOX (capecitabine, oxaliplatin; also called CAPOX), has been the standard adjuvant treatment for stage III colon cancer patients who have good performance status and are able to tolerate aggressive combination chemotherapy. However, the administration of oxaliplatin-containing adjuvant chemotherapy for 6 months is associated with several important toxicities, including myelosuppression, gastrointestinal toxicity in the form of mucositis and/or diarrhea, and neurotoxicity. In particular, the oxaliplatin-induced, cumulative, dose-dependent neurotoxicity, which is usually manifested as a peripheral sensory neuropathy, can be a significant issue for patients.

The current knowledge is based on clinical trials' results (or meta-analyses) that include by definition selected patients e.g. elderly patients. Previous studies mainly explored prognostic factors for DFS and OS, but it is as yet unclear if these factors can predict common toxicity outcomes. Also in Egypt we have lake of data regarding chemotherapy toxicity in colorectal carcinoma patients. The present work was aimed to assess and evaluate chemotherapy toxicities in adjuvant treatment in CRC patients and analyze certain factors that might increase chemotherapy toxicity. 


\section{Patients and Methods}

This was a retrospective where data obtained from medical records of patients with colorectal cancer treated in adjuvant setting between Jan. 2012 and Jan. 2017 in Ain Shams University Oncology Department.

A total of 72 eligible patients diagnosed with histopathologically confirmed carcinoma of colorectal. The mean age was $50.86 \pm 12.97$, with range from 18 to 80 years. $58.3 \%$ were females and $41.7 \%$ were males. $58.3 \%$ diagnosed as colon cancer $41.7 \%$ were rectal cancer. $69.4 \%$ received FOLFOX, 16.7\% received XELOX, $11.1 \%$ received capcitabine and only $2.8 \%$ received de Gramont protocol.

The inclusion criteria were patients with histopathology Diagnosis of colorectal cancer without distant metastasis who were treated by adjuvant chemotherapy at oncology department, adequate hemoglobin level, white blood count and platelet before therapy, ages 18 - 80 years, eastern cooperative oncology group (ECOG) performance score 0 - 2 and acceptable liver and renal function tests.

Patients were excluded from the study if they had undergone neo-adjuvant chemotherapy, if they have distant metastasis, patients with prior malignancy and pregnant patients were also excluded.

All adverse effects were recorded according to common terminology criteria of adverse events (CTCAE) version 5.0 grading scale on a scale of 1 to 5 according to the general grade definition. Evaluations of hematological toxicity, hepatotoxicity and nephrotoxicity were performed on the basis of available laboratory test findings. The activity of the hematopoietic system will be evaluated on the basis of peripheral blood hemoglobin, WBC, granulocyte and thrombocyte count; the activity of the liver will be evaluated on the basis of the levels of bilirubin and liver enzymes activity. Renal function will be assessed on the basis of serum creatinine and urea levels. The tests were performed on a routine basis on the day of the patient's admission to the Chemotherapy Department, i.e. prior to the planned administration of a therapeutic cycle. The neurological, dermatological, cardiac and other toxicities to individual organs and systems will be evaluated on the basis of the patient's own reports.

Data were collected, revised, coded and entered to the statistical package for social science (SPSS) version 22. Qualitative data were presented as numbers and percentages while quantitative data were presented as mean, standard deviations and ranges. The comparison between groups regarding qualitative data was done by using Chi-square test and/or Fisher exact test only when the expected count in any cell found less than 5. The confidence interval was set to $95 \%$ and the margin of Error accepted was set to $5 \%$. So, the p-value was considered significant as the following:

- $\mathrm{p}>0.05$ : Non-significant (NS);

- $\mathrm{p}<0.05$ : Significant (S);

- $\mathrm{p}<0.01$ : Highly significant (HS).

\section{Results}

This was a retrospective where data obtained from medical records of patients 
with colorectal cancer treated in adjuvant setting between Jan. 2012 and Jan. 2017 in Ain shams University Oncology Department.

A total of 72 eligible patients diagnosed with histopathologically confirmed carcinoma of colorectal. The mean age was $50.86 \pm 12.97$, with range from 18 to 80 years. $58.3 \%$ were females and $41.7 \%$ were males. $58.3 \%$ diagnosed as colon cancer, 41.7 \% were rectal cancer. $69.4 \%$ received FOLFOX, 16.7\% received XELOX, $11.1 \%$ received capcitabine and only $2.8 \%$ received de Gramont protocol.

We found that the most chemotherapy reported was neurological toxicity in (73.6\%), gastrointestinal symptoms (diarrhea $52.7 \%$, nausea $30.6 \%$, vomiting $27.8 \%$ \& oral mucositis $24 \%$ ), hematological toxicity (neutropenia $40.3 \%$, anemia $34.7 \%$, thrombocytopenia $12.5 \%$ ), fatigue $20.9 \%$, hepatic toxicity $18.1 \%$, dermatological toxicity $9.7 \%$ \& renal toxicity $5.6 \%$ ) (Table 1 ). Older patients have significant incidence of neurological toxicity $(p$-value $=0.023)$ and fatigue $(p$-value $=$ $0.038)$ (Table 2). Females have significant incidence of anemia $(p$-value $=0.017)$ (Table 3). Increase of oxaliplatin cumulative dose increase incidence of neurological toxicity ( $\mathrm{p}$-value 0.024$)$, thrombocytopenia $(\mathrm{p}$-value $=0.007)$ and renal toxicity $(\mathrm{p}$-value $=0.030)($ Table 4$)$. Oxaliplatin containing regimens have high significant correlation with neurological toxicity ( $\mathrm{p}$-value $=0.000$ ) and capcitabine has high significant correlation with dermatological toxicity $(\mathrm{p}$-value $=0.000)$ (Table 5).

Table 1. Descriptive statistical analysis of all recorded toxicities.

\begin{tabular}{|c|c|c|c|}
\hline & & No. & $\%$ \\
\hline & No & 19 & $26.4 \%$ \\
\hline \multirow[t]{3}{*}{ Neurological toxicity } & $\mathrm{G} 3,4$ & 5 & $6.9 \%$ \\
\hline & $\mathrm{G} 1,2$ & 48 & $66.7 \%$ \\
\hline & No & 47 & $65.3 \%$ \\
\hline \multirow[t]{3}{*}{ Anemia } & $\mathrm{G} 3,4$ & 2 & $2.8 \%$ \\
\hline & $\mathrm{G} 1,2$ & 23 & $31.9 \%$ \\
\hline & No & 63 & $87.5 \%$ \\
\hline \multirow[t]{3}{*}{ Thrombocytopenia } & $\mathrm{G} 3,4$ & 0 & $0.0 \%$ \\
\hline & $\mathrm{G} 1,2$ & 9 & $12.5 \%$ \\
\hline & No & 43 & $59.7 \%$ \\
\hline \multirow[t]{3}{*}{ Neutropenia } & $\mathrm{G} 3,4$ & 12 & $16.7 \%$ \\
\hline & $\mathrm{G} 1,2$ & 17 & $23.6 \%$ \\
\hline & No & 65 & $90.3 \%$ \\
\hline \multirow[t]{2}{*}{ Dermatological toxicity } & $\mathrm{G} 3,4$ & 1 & $1.4 \%$ \\
\hline & $\mathrm{G} 1,2$ & 6 & $8.3 \%$ \\
\hline
\end{tabular}




\section{Continued}

\begin{tabular}{|c|c|c|c|}
\hline & No & 50 & $69.4 \%$ \\
\hline \multirow[t]{3}{*}{ Nausea } & $\mathrm{G} 3,4$ & 1 & $1.4 \%$ \\
\hline & $\mathrm{G} 1,2$ & 21 & $29.2 \%$ \\
\hline & No & 52 & $72.2 \%$ \\
\hline \multirow[t]{3}{*}{ Vomiting } & $\mathrm{G} 3,4$ & 0 & $0.0 \%$ \\
\hline & $\mathrm{G} 1,2$ & 20 & $27.8 \%$ \\
\hline & No & 34 & $47.2 \%$ \\
\hline \multirow[t]{3}{*}{ Diarrhea } & $\mathrm{G} 3,4$ & 5 & $6.9 \%$ \\
\hline & $\mathrm{G} 1,2$ & 33 & $45.8 \%$ \\
\hline & No & 71 & $98.6 \%$ \\
\hline \multirow[t]{3}{*}{ Allergy } & $\mathrm{G} 3,4$ & 0 & $0.0 \%$ \\
\hline & $\mathrm{G} 1,2$ & 1 & $1.4 \%$ \\
\hline & No & 59 & $81.9 \%$ \\
\hline \multirow[t]{3}{*}{ Hepatic toxicity } & $\mathrm{G} 3,4$ & 0 & $0.0 \%$ \\
\hline & $\mathrm{G} 1,2$ & 13 & $18.1 \%$ \\
\hline & No & 68 & $94.4 \%$ \\
\hline \multirow[t]{3}{*}{ Renal toxicity } & $\mathrm{G} 3,4$ & 0 & $0.0 \%$ \\
\hline & $\mathrm{G} 1,2$ & 4 & $5.6 \%$ \\
\hline & No & 72 & $100.0 \%$ \\
\hline \multirow[t]{3}{*}{ Cardiac toxicity } & G3,4 & 0 & $0.0 \%$ \\
\hline & $\mathrm{G} 1,2$ & 0 & $0.0 \%$ \\
\hline & No & 72 & $100.0 \%$ \\
\hline \multirow[t]{3}{*}{ Hair loss } & $\mathrm{G} 3,4$ & 0 & $0.0 \%$ \\
\hline & $\mathrm{G} 1,2$ & 0 & $0.0 \%$ \\
\hline & No & 55 & $76.4 \%$ \\
\hline \multirow[t]{3}{*}{ Oral mucositis } & $\mathrm{G} 3,4$ & 4 & $5.6 \%$ \\
\hline & $\mathrm{G} 1,2$ & 13 & $18.1 \%$ \\
\hline & No & 57 & $79.2 \%$ \\
\hline \multirow[t]{4}{*}{ Fatigue } & $\mathrm{G} 3,4$ & 3 & $4.2 \%$ \\
\hline & $\mathrm{G} 1,2$ & 12 & $16.7 \%$ \\
\hline & Headache & 1 & $1.4 \%$ \\
\hline & Thrombophlebitis G1 & 5 & $6.9 \%$ \\
\hline \multirow[t]{3}{*}{ Others } & Abdominal cramp & 6 & $8.3 \%$ \\
\hline & Phlebitis & 0 & $0.0 \%$ \\
\hline & Abdominal pain G2 & 2 & $2.8 \%$ \\
\hline
\end{tabular}


Table 2. Correlation between age and toxicity.

\begin{tabular}{|c|c|c|c|c|c|c|c|c|}
\hline & & \multicolumn{2}{|c|}{ Age $<50$} & \multicolumn{2}{|c|}{ Age $\geq 50$} & \multirow{2}{*}{ Test value } & \multirow{2}{*}{$\mathrm{p}$-value } & \multirow{2}{*}{ Sig. } \\
\hline & & No. & $\%$ & No. & $\%$ & & & \\
\hline \multirow{3}{*}{$\begin{array}{c}\text { Neurological } \\
\text { toxicity }\end{array}$} & No & 12 & $40.0 \%$ & 7 & $16.7 \%$ & & & \\
\hline & $\mathrm{G} 3,4$ & 0 & $0.0 \%$ & 5 & $11.9 \%$ & 7.525 & 0.023 & S \\
\hline & $\mathrm{G} 1,2$ & 18 & $60.0 \%$ & 30 & $71.4 \%$ & & & \\
\hline \multirow{3}{*}{ Anemia } & No & 20 & $66.7 \%$ & 27 & $64.3 \%$ & & & \\
\hline & $\mathrm{G} 3,4$ & 1 & $3.3 \%$ & 1 & $2.4 \%$ & 0.133 & 0.936 & NS \\
\hline & $\mathrm{G} 1,2$ & 9 & $30.0 \%$ & 14 & $33.3 \%$ & & & \\
\hline \multirow{3}{*}{ Thrombocytopenia } & No & 27 & $90.0 \%$ & 36 & $85.7 \%$ & & & \\
\hline & $\mathrm{G} 3,4$ & 0 & $0.0 \%$ & 0 & $0.0 \%$ & 0.294 & 0.588 & NS \\
\hline & $\mathrm{G} 1,2$ & 3 & $10.0 \%$ & 6 & $14.3 \%$ & & & \\
\hline \multirow{3}{*}{ Neutropenia } & No & 21 & $70.0 \%$ & 22 & $52.4 \%$ & & & \\
\hline & $\mathrm{G} 3,4$ & 5 & $16.7 \%$ & 7 & $16.7 \%$ & 3.210 & 0.201 & NS \\
\hline & $\mathrm{G} 1,2$ & 4 & $13.3 \%$ & 13 & $31.0 \%$ & & & \\
\hline \multirow{3}{*}{$\begin{array}{l}\text { Dermatological } \\
\text { toxicity }\end{array}$} & No & 25 & $83.3 \%$ & 40 & $95.2 \%$ & & & \\
\hline & $\mathrm{G} 3,4$ & 1 & $3.3 \%$ & 0 & $0.0 \%$ & 3.218 & 0.200 & NS \\
\hline & $\mathrm{G} 1,2$ & 4 & $13.3 \%$ & 2 & $4.8 \%$ & & & \\
\hline \multirow{3}{*}{ Nausea } & No & 23 & $76.7 \%$ & 27 & $64.3 \%$ & & & \\
\hline & $\mathrm{G} 3,4$ & 0 & $0.0 \%$ & 1 & $2.4 \%$ & 1.701 & 0.427 & NS \\
\hline & $\mathrm{G} 1,2$ & 7 & $23.3 \%$ & 14 & $33.3 \%$ & & & \\
\hline \multirow{3}{*}{ Vomiting } & No & 22 & $73.3 \%$ & 30 & $71.4 \%$ & & & \\
\hline & $\mathrm{G} 3,4$ & 0 & $0.0 \%$ & 0 & $0.0 \%$ & 0.032 & 0.859 & NS \\
\hline & $\mathrm{G} 1,2$ & 8 & $26.7 \%$ & 12 & $28.6 \%$ & & & \\
\hline \multirow{3}{*}{ Diarhea } & No & 14 & $46.7 \%$ & 20 & $47.6 \%$ & & & \\
\hline & $\mathrm{G} 3,4$ & 3 & $10.0 \%$ & 2 & $4.8 \%$ & 0.765 & 0.682 & NS \\
\hline & $\mathrm{G} 1,2$ & 13 & $43.3 \%$ & 20 & $47.6 \%$ & & & \\
\hline \multirow{3}{*}{ Allergy } & No & 29 & $96.7 \%$ & 42 & $100.0 \%$ & & & \\
\hline & $\mathrm{G} 3,4$ & 0 & $0.0 \%$ & 0 & $0.0 \%$ & 1.420 & 0.233 & NS \\
\hline & $\mathrm{G} 1,2$ & 1 & $3.3 \%$ & 0 & $0.0 \%$ & & & \\
\hline \multirow{3}{*}{ Hepatic toxicity } & No & 22 & $73.3 \%$ & 37 & $88.1 \%$ & & & \\
\hline & $\mathrm{G} 3,4$ & 0 & $0.0 \%$ & 0 & $0.0 \%$ & 2.577 & 0.108 & NS \\
\hline & $\mathrm{G} 1,2$ & 8 & $26.7 \%$ & 5 & $11.9 \%$ & & & \\
\hline \multirow{3}{*}{ Renal toxicity } & No & 28 & $93.3 \%$ & 40 & $95.2 \%$ & & & \\
\hline & $\mathrm{G} 3,4$ & 0 & $0.0 \%$ & 0 & $0.0 \%$ & 0.121 & 0.728 & NS \\
\hline & $\mathrm{G} 1,2$ & 2 & $6.7 \%$ & 2 & $4.8 \%$ & & & \\
\hline \multirow{3}{*}{ Oral mucositis } & No & 26 & $86.7 \%$ & 29 & $69.0 \%$ & & & \\
\hline & $\mathrm{G} 3,4$ & 1 & $3.3 \%$ & 3 & $7.1 \%$ & 3.017 & 0.221 & NS \\
\hline & $\mathrm{G} 1,2$ & 3 & $10.0 \%$ & 10 & $23.8 \%$ & & & \\
\hline \multirow{3}{*}{ Fatigue } & No & 28 & $93.3 \%$ & 29 & $69.0 \%$ & & & \\
\hline & $\mathrm{G} 3,4$ & 0 & $0.0 \%$ & 3 & $7.1 \%$ & 6.532 & 0.038 & S \\
\hline & $\mathrm{G} 1,2$ & 2 & $6.7 \%$ & 10 & $23.8 \%$ & & & \\
\hline \multirow{5}{*}{ Others } & Headache & 1 & $3.3 \%$ & 0 & $0.0 \%$ & & & \\
\hline & Thrombophlebitis & 2 & $6.7 \%$ & 3 & $7.1 \%$ & & & \\
\hline & Abdominal cramp & 1 & $3.3 \%$ & 5 & $11.9 \%$ & 3.384 & 0.336 & NS \\
\hline & Phlebitis & 0 & $0.0 \%$ & 0 & $0.0 \%$ & & & \\
\hline & Abdominal pain & 0 & $0.0 \%$ & 2 & $4.8 \%$ & & & \\
\hline
\end{tabular}


Table 3. Correlation between gender and toxicity.

\begin{tabular}{|c|c|c|c|c|c|c|c|c|}
\hline & & \multicolumn{2}{|c|}{ Female } & \multicolumn{2}{|c|}{ Male } & \multirow{2}{*}{ Test value } & \multirow{2}{*}{ p-value } & \multirow{2}{*}{ Sig } \\
\hline & & No. & $\%$ & No. & $\%$ & & & \\
\hline \multirow{3}{*}{$\begin{array}{c}\text { Neurological } \\
\text { toxicity }\end{array}$} & No & 9 & $21.4 \%$ & 10 & $33.3 \%$ & & & \\
\hline & $\mathrm{G} 3,4$ & 5 & $11.9 \%$ & 0 & $0.0 \%$ & 4.511 & 0.105 & NS \\
\hline & $\mathrm{G} 1,2$ & 28 & $66.7 \%$ & 20 & $66.7 \%$ & & & \\
\hline \multirow{3}{*}{ Anemia } & No & 22 & $52.4 \%$ & 25 & $83.3 \%$ & & & \\
\hline & $\mathrm{G} 3,4$ & 1 & $2.4 \%$ & 1 & $3.3 \%$ & 8.202 & 0.017 & $S$ \\
\hline & $\mathrm{G} 1,2$ & 19 & $45.2 \%$ & 4 & $13.3 \%$ & & & \\
\hline \multirow{3}{*}{ Thrombocytopenia } & No & 36 & $85.7 \%$ & 27 & $90.0 \%$ & & & \\
\hline & $\mathrm{G} 3,4$ & 0 & $0.0 \%$ & 0 & $0.0 \%$ & 0.294 & $0.588 \mathrm{~b}$ & NS \\
\hline & $\mathrm{G} 1,2$ & 6 & $14.3 \%$ & 3 & $10.0 \%$ & & & \\
\hline \multirow{3}{*}{ Neutropenia } & No & 21 & $50.0 \%$ & 22 & $73.3 \%$ & & & \\
\hline & $\mathrm{G} 3,4$ & 10 & $23.8 \%$ & 2 & $6.7 \%$ & 4.965 & 0.084 & NS \\
\hline & $\mathrm{G} 1,2$ & 11 & $26.2 \%$ & 6 & $20.0 \%$ & & & \\
\hline \multirow{3}{*}{$\begin{array}{l}\text { Dermatological } \\
\text { toxicity }\end{array}$} & No & 39 & $92.9 \%$ & 26 & $86.7 \%$ & & & \\
\hline & $\mathrm{G} 3,4$ & 0 & $0.0 \%$ & 1 & $3.3 \%$ & 1.646 & 0.439 & NS \\
\hline & $\mathrm{G} 1,2$ & 3 & $7.1 \%$ & 3 & $10.0 \%$ & & & \\
\hline \multirow{3}{*}{ Nausea } & No & 30 & $71.4 \%$ & 20 & $66.7 \%$ & & & \\
\hline & $\mathrm{G} 3,4$ & 1 & $2.4 \%$ & 0 & $0.0 \%$ & 1.078 & 0.583 & NS \\
\hline & $\mathrm{G} 1,2$ & 11 & $26.2 \%$ & 10 & $33.3 \%$ & & & \\
\hline \multirow{3}{*}{ Vomiting } & No & 29 & $69.0 \%$ & 23 & $76.7 \%$ & & & \\
\hline & $\mathrm{G} 3,4$ & 0 & $0.0 \%$ & 0 & $0.0 \%$ & 0.506 & 0.477 & NS \\
\hline & $\mathrm{G} 1,2$ & 13 & $31.0 \%$ & 7 & $23.3 \%$ & & & \\
\hline \multirow{3}{*}{ Diarrhea } & No & 16 & $38.1 \%$ & 18 & $60.0 \%$ & & & \\
\hline & $\mathrm{G} 3,4$ & 2 & $4.8 \%$ & 3 & $10.0 \%$ & 5.283 & 0.071 & NS \\
\hline & $\mathrm{G} 1,2$ & 24 & $57.1 \%$ & 9 & $30.0 \%$ & & & \\
\hline \multirow{3}{*}{ Allergy } & No & 41 & $97.6 \%$ & 30 & $100.0 \%$ & & & \\
\hline & $\mathrm{G} 3,4$ & 0 & $0.0 \%$ & 0 & $0.0 \%$ & 0.724 & 0.395 & NS \\
\hline & $\mathrm{G} 1,2$ & 1 & $2.4 \%$ & 0 & $0.0 \%$ & & & \\
\hline \multirow{3}{*}{ Hepatic toxicity } & No & 34 & $81.0 \%$ & 25 & $83.3 \%$ & & & \\
\hline & $\mathrm{G} 3,4$ & 0 & $0.0 \%$ & 0 & $0.0 \%$ & 0.067 & 0.796 & NS \\
\hline & $\mathrm{G} 1,2$ & 8 & $19.0 \%$ & 5 & $16.7 \%$ & & & \\
\hline \multirow{3}{*}{ Renal toxicity } & No & 38 & $90.5 \%$ & 30 & $100.0 \%$ & & & \\
\hline & $\mathrm{G} 3,4$ & 0 & $0.0 \%$ & 0 & $0.0 \%$ & 3.025 & 0.082 & NS \\
\hline & $\mathrm{G} 1,2$ & 4 & $9.5 \%$ & 0 & $0.0 \%$ & & & \\
\hline \multirow{3}{*}{ Oral mucositis } & No & 30 & $71.4 \%$ & 25 & $83.3 \%$ & & & \\
\hline & $\mathrm{G} 3,4$ & 4 & $9.5 \%$ & 0 & $0.0 \%$ & 3.237 & 0.198 & NS \\
\hline & $\mathrm{G} 1,2$ & 8 & $19.0 \%$ & 5 & $16.7 \%$ & & & \\
\hline \multirow{3}{*}{ Fatigue } & No & 31 & $73.8 \%$ & 26 & $86.7 \%$ & & & \\
\hline & $\mathrm{G} 3,4$ & 2 & $4.8 \%$ & 1 & $3.3 \%$ & 1.823 & 0.402 & NS \\
\hline & $\mathrm{G} 1,2$ & 9 & $21.4 \%$ & 3 & $10.0 \%$ & & & \\
\hline \multirow{5}{*}{ Others } & Headache & 1 & $1 \%$ & 0 & $0.0 \%$ & & & \\
\hline & Thrombophlebitis & 2 & $4.8 \%$ & 3 & $10.0 \%$ & & & \\
\hline & Abdominal cramp & 3 & $7.1 \%$ & 3 & $10.0 \%$ & 2.315 & 0.510 & NS \\
\hline & Phlebitis & 0 & $0.0 \%$ & 0 & $0.0 \%$ & & & \\
\hline & Abdominal pain & 2 & $4.8 \%$ & 0 & $0.0 \%$ & & & \\
\hline
\end{tabular}


N. S. Gobran et al.

Table 4. Correlation between oxaliplatin cumulative dose and toxicity.

\begin{tabular}{|c|c|c|c|c|c|c|c|c|}
\hline & & \multicolumn{2}{|c|}{ Dose $<1400$} & \multicolumn{2}{|c|}{ Dose $\geq 1400$} & \multirow{2}{*}{ Test value } & \multirow{2}{*}{ p-value } & \multirow{2}{*}{ Sig } \\
\hline & & No. & $\%$ & No. & $\%$ & & & \\
\hline \multirow{3}{*}{$\begin{array}{c}\text { Neurological } \\
\text { toxicity }\end{array}$} & No & 13 & $34.2 \%$ & 6 & $17.6 \%$ & & & \\
\hline & $\mathrm{G} 3,4$ & 0 & $0.0 \%$ & 5 & $14.7 \%$ & 7.463 & 0.024 & S \\
\hline & $\mathrm{G} 1,2$ & 25 & $65.8 \%$ & 23 & $67.6 \%$ & & & \\
\hline \multirow{3}{*}{ Anemia } & No & 28 & $73.7 \%$ & 19 & $55.9 \%$ & & & \\
\hline & $\mathrm{G} 3,4$ & 0 & $0.0 \%$ & 2 & $5.9 \%$ & 3.905 & 0.142 & NS \\
\hline & $\mathrm{G} 1,2$ & 10 & $26.3 \%$ & 13 & $38.2 \%$ & & & \\
\hline \multirow{3}{*}{ Thrombocytopenia } & No & 37 & $97.4 \%$ & 26 & $76.5 \%$ & & & \\
\hline & $\mathrm{G} 3,4$ & 0 & $0.0 \%$ & 0 & $0.0 \%$ & 7.165 & 0.007 & HS \\
\hline & $\mathrm{G} 1,2$ & 1 & $2.6 \%$ & 8 & $23.5 \%$ & & & \\
\hline \multirow{3}{*}{ Neutropenia } & No & 23 & $60.5 \%$ & 20 & $58.8 \%$ & & & \\
\hline & $\mathrm{G} 3,4$ & 7 & $18.4 \%$ & 5 & $14.7 \%$ & 0.380 & 0.827 & NS \\
\hline & $\mathrm{G} 1,2$ & 8 & $21.1 \%$ & 9 & $26.5 \%$ & & & \\
\hline \multirow{3}{*}{$\begin{array}{l}\text { Dermatological } \\
\text { toxicity }\end{array}$} & No & 33 & $86.8 \%$ & 32 & $94.1 \%$ & & & \\
\hline & $\mathrm{G} 3,4$ & 1 & $2.6 \%$ & 0 & $0.0 \%$ & 1.464 & 0.481 & NS \\
\hline & G1,2 & 4 & $10.5 \%$ & 2 & $5.9 \%$ & & & \\
\hline \multirow{3}{*}{ Nausea } & No & 26 & $68.4 \%$ & 24 & $70.6 \%$ & & & \\
\hline & $\mathrm{G} 3,4$ & 0 & $0.0 \%$ & 1 & $2.9 \%$ & 1.290 & 0.525 & NS \\
\hline & G1,2 & 12 & $31.6 \%$ & 9 & $26.5 \%$ & & & \\
\hline \multirow{3}{*}{ Vomiting } & No & 25 & $65.8 \%$ & 27 & $79.4 \%$ & & & \\
\hline & $\mathrm{G} 3,4$ & 0 & $0.0 \%$ & 0 & $0.0 \%$ & 1.660 & 0.198 & NS \\
\hline & $\mathrm{G} 1,2$ & 13 & $34.2 \%$ & 7 & $20.6 \%$ & & & \\
\hline \multirow{3}{*}{ Diarhea } & No & 17 & $44.7 \%$ & 17 & $50.0 \%$ & & & \\
\hline & $\mathrm{G} 3,4$ & 4 & $10.5 \%$ & 1 & $2.9 \%$ & 1.613 & 0.446 & NS \\
\hline & G1,2 & 17 & $44.7 \%$ & 16 & $47.1 \%$ & & & \\
\hline \multirow{3}{*}{ Allergy } & No & 37 & $97.4 \%$ & 34 & $100.0 \%$ & & & \\
\hline & $\mathrm{G} 3,4$ & 0 & $0.0 \%$ & 0 & $0.0 \%$ & 0.907 & 0.341 & NS \\
\hline & G1,2 & 1 & $2.6 \%$ & 0 & $0.0 \%$ & & & \\
\hline \multirow{3}{*}{ Hepatic toxicity } & No & 31 & $81.6 \%$ & 28 & $82.4 \%$ & & & \\
\hline & $\mathrm{G} 3,4$ & 0 & $0.0 \%$ & 0 & $0.0 \%$ & 0.007 & 0.932 & NS \\
\hline & G1,2 & 7 & $18.4 \%$ & 6 & $17.6 \%$ & & & \\
\hline \multirow{3}{*}{ Renal toxicity } & No & 38 & $100.0 \%$ & 30 & $88.2 \%$ & & & \\
\hline & $\mathrm{G} 3,4$ & 0 & $0.0 \%$ & 0 & $0.0 \%$ & 4.734 & 0.030 & $S$ \\
\hline & G1,2 & 0 & $0.0 \%$ & 4 & $11.8 \%$ & & & \\
\hline \multirow{3}{*}{ Oral mucositis } & No & 28 & $73.7 \%$ & 27 & $79.4 \%$ & & & \\
\hline & $\mathrm{G} 3,4$ & 3 & $7.9 \%$ & 1 & $2.9 \%$ & 0.876 & 0.645 & NS \\
\hline & $\mathrm{G} 1,2$ & 7 & $18.4 \%$ & 6 & $17.6 \%$ & & & \\
\hline \multirow{3}{*}{ Fatigue } & No & 32 & $84.2 \%$ & 25 & $73.5 \%$ & & & \\
\hline & $\mathrm{G} 3,4$ & 1 & $2.6 \%$ & 2 & $5.9 \%$ & 1.308 & 0.520 & NS \\
\hline & G1,2 & 5 & $13.2 \%$ & 7 & $20.6 \%$ & & & \\
\hline \multirow{5}{*}{ Others } & Headache & 0 & $0.0 \%$ & 1 & $2.9 \%$ & & & \\
\hline & Thrombophlebitis & 1 & $2.6 \%$ & 4 & $11.8 \%$ & & & \\
\hline & Abdominal cramp & 1 & $2.6 \%$ & 5 & $14.7 \%$ & 6.315 & 0.097 & NS \\
\hline & Phlebitis & 0 & $0.0 \%$ & 0 & $0.0 \%$ & & & \\
\hline & Abdominal pain & 1 & $2.6 \%$ & 1 & $2.9 \%$ & & & \\
\hline
\end{tabular}


N. S. Gobran et al.

Table 5. Correlation between type of chemotherapy and toxicity.

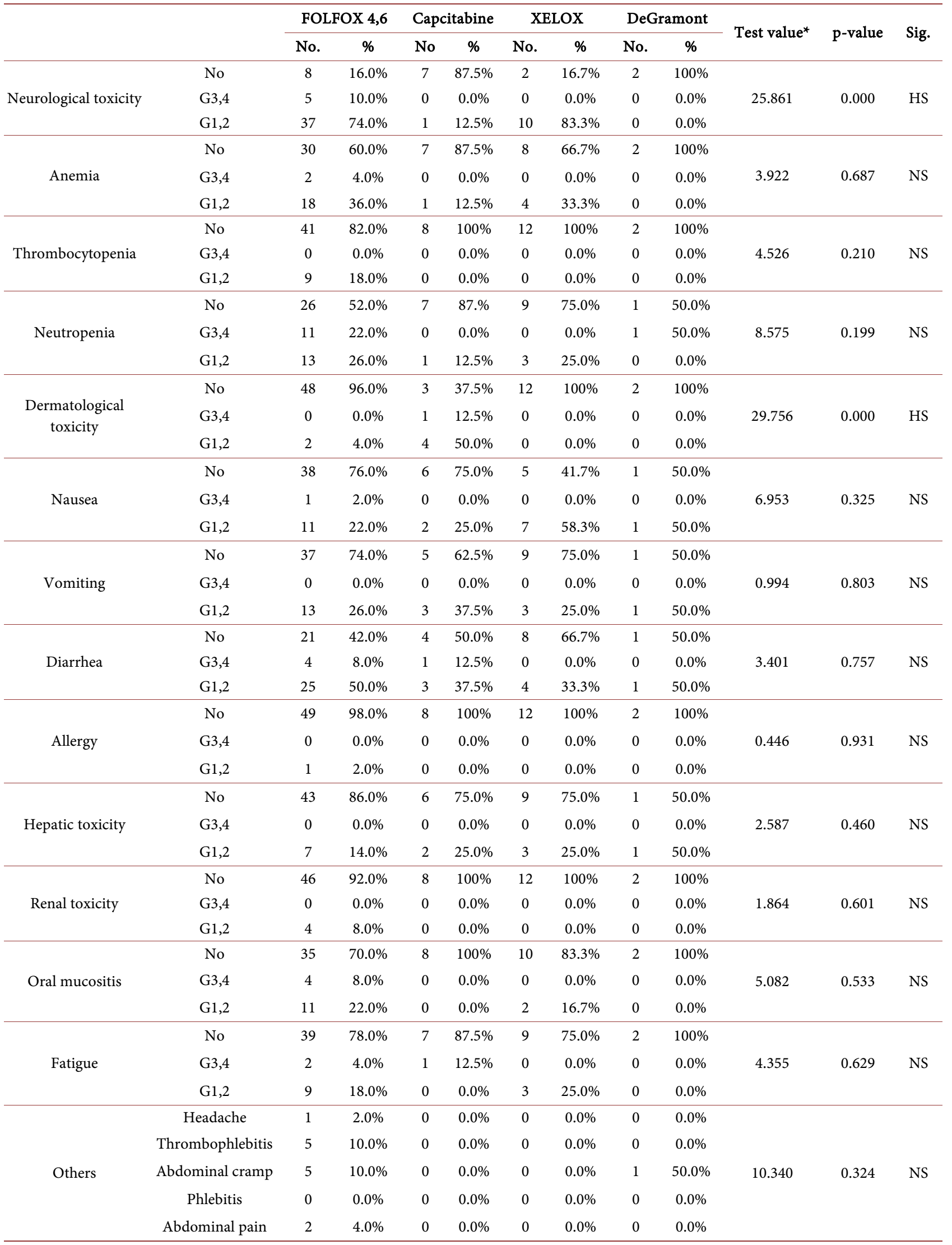




\section{Discussion}

In our study we recorded different adverse events occurred with different chemotherapy regimens received in adjuvant treated colorectal carcinoma patients in Ain Shams University Oncology Department. We also make comparison between toxicities in oxaliplatin based regimens (FOLFOX and XELOX) and monotherapy as capcitabine and attempt to explore the association between certain factors and different toxicities.

The mean age of patients in our study was 50.86 years. $58.3 \%$ were females and $41.7 \%$ were males. $58.3 \%$ diagnosed as colon cancer $41.7 \%$ were rectal cancer. $69.4 \%$ received FOLFOX, 16.7\% received XELOX, 11.1\% received capcitabine and only $2.8 \%$ received de Gramont protocol.

In our study the most common adverse events in adjuvant treated colorectal cancer patient was neurological toxicity $73.6 \%$, diarrhea $52.7 \%$, neutropenia $40.3 \%$, anemia $34.7 \%$, nausea $30.6 \%$ and vomiting $27.8 \%$. these finding are consistent with finding in mosaic trial [6] which found that $92 \%$ of patients received oxaliplatin have neurological toxicity, $78 \%$ neutropenia, $75.6 \%$ anemia, $73.7 \%$ nausea, $56 \%$ diarrhea and $47 \%$ vomiting. They are also in agreement with the finding published by Jeon et al. in 2011 [7] which found that neurological toxicity $81.7 \%$, neutropenia $74.7 \%$, diarrhea $65 \%$ nausea $62.2 \%$, anemia $46.3 \%$ and vomiting $14.6 \%$.

In comparison between different chemotherapy regimens we found that the most common toxicity occurred in patients received capecitabine monotherapy was dermatological toxicity (hand foot syndrome) $62.5 \%$ and this shows high statistical significance. The second most common toxicity was gastrointestinal symptoms in the form of diarrhea $50 \%$, vomiting $37.5 \%$ nausea $25 \%$ then comes hepatic toxicity occurred in $25 \%$ of patients received capecitabine as monotherapy and then hematological toxicity (anemia 12.5\% and neutropenia 12.5\%) this results largely consists with findings from previous study by Chris twelves et al. (X-ACT trial) [8] which found that the most common toxicity in patients received capecitabine as monotherapy in stage iii colon cancer was HFS $60 \%$, hepatic toxicity $50 \%$, diarrhea $46 \%$, nausea and vomiting $36 \%$ and neutropenia $32 \%$. this also consistent with findings from a previous study by Ardavanis et al. [9] which found that $89 \%$, $75 \%$, and $79 \%$ of patients had HFS, diarrhea, and anemia, respectively, but less than a quarter of these cases were grade III in severity.

In oxaliplatin based chemotherapy regimens (FOLFOX \& XELOX) the most common toxicity in our study was neurological toxicity $84 \%$ of patients received FOLFOX and $83 \%$ of patients received XELOX and this showed high statistical significance in correlation to other regimens this is largely consistent with mosaic trial [6] that found that $92 \%$ of patient received oxaliplatin suffered from peripheral neuropathy. These are also in agreement with findings published by Jeon et al. in 2011 [7] which found that neurological toxicity occurred in $81.7 \%$ of patients received FOLFOX regimen.

In comparison between FOLFOX adverse events and XELOX adverse events our study found that patients received FOLFOX regimen had more incidence of neutropenia $46 \%$ (G i,ii, $24 \%$ and G iii,iv, $22 \%$ ) while only $25 \%$ of patients re- 
ceived XELOX suffered from neutropenia all of them was grade $1 \& 2$ these finding agree with randomized phase 3 study of Capecitabine plus Oxaliplatin Compared with Fluorouracil/Folinic Acid plus Oxaliplatin as First-Line Therapy for Metastatic Colorectal Cancer conducted by Jim Cassidy et al. [10] that showed that the rates of some individual adverse events differed between the two regimens. Whereas FOLFOX was associated with more grade 3/4 neutropenia/granulocytopenia ( $44 \%$ vs $7 \%$ ).

In our study we attempted to explore the association between some factors and the toxicity outcomes of CRC patients treated with adjuvant systemic therapy. We found that older patient ( $\geq 50$ years) have significant increase ( $\mathrm{p}$-value $=$ 0.023 ) in the incidence of neurological toxicity. This is not consistent with the study done by Goldberg et al. [11] which showed age alone does not appear to be a strong factor influencing neurological toxicity outcomes. A retrospective analysis has been performed of 3742 patients, including 614 aged over 70 years, who received 5FU/oxaliplatin chemotherapy in the adjuvant or advanced settings. This demonstrated a modest increase in the rate of significant hematological toxicity, but similar rates of non-hematological toxicity in patients over 70 years compared with younger patients. Elderly patients also had similar survival outcomes.

In our study age also showed statistical significance $(p$-value $=0.038)$ with the occurrence of fatigue. Older patient suffers from fatigue during chemotherapy more than younger patients this is consistent with Goldberg et al. [11] which confirmed that Fatigue emerged as a statistically significant difference between the age groups when analyzed using age as a continuous rather than dichotomous variable and this event generally reverses at therapy cessation.

One of the objectives of our study was to find the impact of gender in toxicity outcomes in adjuvant treated CRC patients. We found that females statistically significant ( $\mathrm{p}=0.017$ ) have more incidence of anemia. This consistent with the study by Omar Abdelrahman [12] the study provides an assessment of the impact of sex on the outcomes of metastatic colorectal cancer receiving first-line systemic therapy. The study showed that when Comparing females to males, females were more likely to have alopecia ( $20 \%$ vs $8.6 \%$; $<<0.001)$, all-grade diarrhea $(60.3 \%$ vs $56.7 \%$; $\mathrm{p}=0.039)$, all-grade nausea and vomiting ( $68.7 \%$ vs $56.6 \%$; $<<0.001)$, high-grade nausea and vomiting $(7.1 \%$ vs $4.5 \% ; \mathrm{p}=0.002)$, all-grade anemia $(19.6 \%$ vs $14.2 \%$; $<<0.001)$, all grade neutropenia $(51.1 \%$ vs $36.6 \%$; $<<0.001)$ and high-grade neutropenia ( $37.1 \%$ vs $24.1 \%$; $\mathrm{p}<0.001$ ).

In our study we tried to find correlation between cumulative dose of oxaliplatin and toxicity outcomes. We found there is significant correlation between oxaliplatin cumulative dose and the severity of neurotoxicity. Our study confirmed that patients received cumulative dose oxaliplatin more than $1400 \mathrm{mg}$ which is the median dose in our study had high grade toxicity $(14.7 \%$ vs $0.0 \%$ p-value $=0.024)$ this results consist with the study conducted by Arun V. Krishnan et al. [13] which confirms the importance of cumulative dose in the development of neuropathy. This also confirmed by the study conducted by Argyriou et al. [14] which showed that the worst severity of acute neurotoxicity was related to the cumulative oxa- 
liplatin dose ( $\mathrm{p}$-value $=0.026)$. Another study confirmed these finding [15] it found that the severity of cumulative OXA-IPN, as graded with both the NCI-CTCv3 ( $\mathrm{r}$ $=0.246 ; \mathrm{p}=0.031)$ and TNSc $(\mathrm{r}=0.228 ; \mathrm{p}=0.046)$, strongly correlated with the cumulative OXA dose during FOLFOX-4 treatment. The same significant correlation emerged between the incidence $(r=0.252 ; \mathrm{p}=0.031)$ and severity $(\mathrm{r}=$ $0.308 ; \mathrm{p}=0.008$ ) of cumulative OXA-IPN during XELOX treatment.

In our study we found that CRC patients received cumulative dose oxaliplatin more than $1400 \mathrm{mg}$ which is the median dose in our study had more incidence of thrombocytopenia (23.5\% vs $2.6 \%$ ) and all thrombocytopenia was grade 1 and 2 in MOSAIC trial [6] the incidence of thrombocytopenia of all grades was $77.4 \%$ (grade $3 \& 4$ only 1.7\%) in FOLFOX arm vs 19\% (grade $3 \& 4$ only $0.4 \%$ ) in $5 \mathrm{FU} / \mathrm{LV}$ arm ( $\mathrm{p}$-value $=0.001$ ).

In a review of chemotherapy-induced thrombocytopenia including $>47,000 \mathrm{pa}-$ tients with solid tumors, CRC was associated with the highest prevalence of thrombocytopenia by cancer type, and most patients had received a platinum-based chemotherapy regimen [16]. Although grades 3 - 4 thrombocytopenia are noted in only $3 \%-4 \%$ of patients exposed to oxaliplatin, this toxicity is of significant concern since the majority of patients with CRC will receive this drug in a given moment of their treatment and the incidence of this toxicity tends to increase during repeated exposures [16].

In our study we found that colorectal cancer patients received adjuvant chemotherapy have low incidence of low grade nephrotoxicity $5.6 \%$. All these patients who have nephrotoxicity received FOLFOX protocol. We found that cumulative dose of oxaliplatin has statistically significant correlation with incidence of nephrotoxicity. Patients received cumulative dose oxaliplatin more than $1400 \mathrm{mg}$ had incidence of nephrotoxicity $11.8 \%$ vs $0.0 \%$ in patients received cumulative dose less than $1400 \mathrm{mg}$ ( $\mathrm{p}$-value $=0.030$ ).

Oxaliplatin nephrotoxicity is rare [17]. In a phase I study of oxaliplatin, a WHO grade 2 transient serum creatinine elevation was observed in $4 \%$ of patients [18]. In recent years, there have been multiple reports of different forms of renal toxicity related to oxaliplatin in combination with other agents [19].

\section{Conclusions}

The most overall toxicity reported during adjuvant treatment in CRC was neurological toxicity. Although a variety of adverse reactions were reported the treatment regimens were tolerated as almost all of patients in the study completed the scheduled chemotherapy course and only one patient stopped chemotherapy due to intolerable toxicity. We also should consider age, sex, type of chemotherapy and cumulative dose of oxaliplatin as predictors for certain toxicities.

There are some limitations in the present study. This retrospective study included an uncontrolled methodology and a limited number of patients from a single cohort. In some patients' files there was no accurate documentation of grade of toxicity. 


\section{Conflicts of Interest}

The authors declare no conflicts of interest regarding the publication of this paper.

\section{References}

[1] Siegel, R.L., Miller, K.D. and Jemal, A. (2018) Cancer Statistics, 2018. CA: A Cancer Journal for Clinicians, 68, 7-30. https://doi.org/10.3322/caac. 21442

[2] Edge, S.B. and Compton, C.C. (2010) The American Joint Committee on Cancer: The 7th Edition of the AJCC Cancer Staging Manual and the Future of TNM. Annals of Surgical Oncology, 17, 1471-1474. https://doi.org/10.1245/s10434-010-0985-4

[3] Chu, E. (2018) Neoplasms of the Small and Large Intestine. In: Goldman, L. and Schafer, A., Eds., Goldman-Cecil Medicine, 26th Edition, Elsevier, New York, in press.

[4] Sargent, D., Sobrero, A., Grothey, A., et al. (2009) Evidence for Cure by Adjuvant Therapy in Colon Cancer: Observations Based on Individual Patient Data from 20,898 Patients on 18 Randomized Trials. Journal of Clinical Oncology, 27, 872-877. https://doi.org/10.1200/JCO.2008.19.5362

[5] Shah, M.A., Renfro, L.A., Allegra, C.J., et al. (2016) Impact of Patient Factors on Recurrence Risk and Time Dependency of Oxaliplatin Benefit in Patients with Colon Cancer: Analysis from Modern-Era Adjuvant Studies in the Adjuvant Colon Cancer end Points (ACCENT) Database. Journal of Clinical Oncology, 34, 843-853. https://doi.org/10.1200/JCO.2015.63.0558

[6] Andre, T., Boni, C., Mounedji-Boudiaf, L., et al. (2004) Oxaliplatin, Fluorouracil, and Leucovorin as Adjuvant Treatment for Colon Cancer. The New England Journal of Medicine, 350, 2343-2351. https://doi.org/10.1056/NEJMoa032709

[7] Jeon, H.-J., Woo, J.-H., Lee, H.-Y., et al. (2011) Adjuvant Chemotherapy Using the FOLFOX Regimen in Colon Cancer. Journal of the Korean Society of Coloproctology, 27, 140-146. https://doi.org/10.3393/jksc.2011.27.3.140

[8] Twelves, C., Wong, A., Nowacki, M.P., et al. (2005) Capecitabine as Adjuvant Treatment for Stage III Colon Cancer. The New England Journal of Medicine, 352 2696-2704. https://doi.org/10.1056/NEJMoa043116

[9] Ardavanis, A.S., Ioannidis, G.N., Orphanos, G.S., et al. (2006) Salvage Treatment with Single-Agent Capecitabine in Patients with Heavily Pretreated Advanced Colorectal Cancer. Anticancer Research, 26, 1669-1672.

[10] Cassidy, J., Clarke, S., Díaz-Rubio, E., et al. (2008) Randomized Phase III Study of Capecitabine plus Oxaliplatin Compared with Fluorouracil/Folinic Acid plus Oxaliplatin as First-Line Therapy for Metastatic Colorectal Cancer. Journal of Clinical Oncology, 26, 2006-2012. https://doi.org/10.1200/JCO.2007.14.9898

[11] Goldberg, R.M., Tabah-Fisch, I., Bleiberg, H., et al. (2006) Pooled Analysis of Safety and Efficacy of Oxaliplatin plus Fluorouracil/Leucovorin Administered Bimonthly in Elderly Patients with Colorectal Cancer. Journal of Clinical Oncology, 24, 4085-4091. https://doi.org/10.1200/JCO.2006.06.9039

[12] Abdel-Rahman, O. (2018) Impact of Sex on Chemotherapy Toxicity and Efficacy among Patients with Metastatic Colorectal Cancer: A Pooled Analysis of Five Randomized Trials. Clinical Colorectal Cancer, 18, 110-115.e2.

https://doi.org/10.1016/j.clcc.2018.12.006 
[13] Krishnan, A.V., Goldstein, D., Friedlander, M., et al. (2005) Oxaliplatin-Induced Neurotoxicity and the Development of Neuropathy. Muscle \& Nerve, 32, 51-60. https://doi.org/10.1002/mus.20340

[14] Argyriou, A.A., Cavaletti, G., Briani, C., et al. (2013) Clinical Pattern and Associations of Oxaliplatin Acute Neurotoxicity: A Prospective Study in 170 Patients with Colorectal Cancer. Cancer, 119, 438-444. https://doi.org/10.1002/cncr.27732

[15] Argyriou, A.A., Velasco, R., Briani, C., et al. (2012) Peripheral Neurotoxicity of Oxaliplatin in Combination with 5-Fluorouracil (FOLFOX) or Capecitabine (XELOX): A Prospective Evaluation of 150 Colorectal Cancer Patients. Annals of Oncology, 23, 3116-3122. https://doi.org/10.1093/annonc/mds208

[16] Wu, Y., Aravind, S., Ranganathan, G., et al. (2009) Anemia and Thrombocytopenia in Patients Undergoing Chemotherapy for Solid Tumors: A Descriptive Study of a Large Outpatient Oncology Practice Database, 2000-2007. Clinical Therapeutics, 31, 2416-2432. https://doi.org/10.1016/j.clinthera.2009.11.020

[17] Haller, D.G. (2000) Safety of Oxaliplatin in the Treatment of Colorectal Cancer. Oncology (Williston Park), 14, 15-20.

[18] Extra, J.M., Marty, M., Brienza, S., et al. (1998) Pharmacokinetics and Safety Profile of Oxaliplatin. Seminars in Oncology, 25, 13-22.

[19] Márquez, E., Rodríguez, E. and Pascual, J. (2013) inadvertent Severe Acute Kidney Injury and Oxaliplatin. International Urology and Nephrology, 45, 297-298. https://doi.org/10.1007/s11255-012-0177-1 